\section{Monolithically integrated DBR waveguide laser and intensity modulator in erbium doped $\mathrm{LiNbO}_{3}$}

J. Söchtig, H. Schütz, R. Widmer, R. Corsini, D. Hiller, C. Carmannini, G. Consonni, S. Bosso and L. Gobbi

Indexing terms: Integrated optics, Distributed Bragg reflector lasers, Waveguide lasers, Lithium niobate

For the first time, a DBR laser has been integrated with a Mach Zehnder intensity modulator in Er diffusion doped, Z cu $\mathrm{LiNbO}_{3}$, with $\mathrm{Ti}$ indiffused waveguides and dry etched Bragg gratings. The monolithically integrated device has a threshold of $54.8 \mathrm{~mW}$ incident pump power $\left(\lambda_{p}=1480 \mathrm{~nm}\right)$ and emits, in a single longitudinal mode, up to $0.65 \mathrm{~mW} \mathrm{CW}$ output power at $\lambda$ s $=1561 \mathrm{~nm}$. The Mach-Zehnder type intensity modulator has an optical bandwidth $>3 \mathrm{GHz}$.

Introduction: Free running lasers, tunable lasers, modelocked lasers and distributed Bragg reflector (DBR) waveguide lasers have all been demonstrated in erbium diffusion doped $\mathrm{LiNbO}_{3}$ $[1-4]$. We report the first singlemode $\mathrm{Er}: \mathrm{LiNbO}_{3} \mathrm{DBR}$ waveguide laser monolithically integrated with a Mach-Zehnder type intensity modulator. This transmitter prototype is an attractive device for high speed analogue transmission and sensor applications. Details of the distributed Bragg reflector (DBR) waveguide laser combined with the Mach-Zehnder type intensity modulator configuration and the basic steps for the formation of the integrated chip are described, followed by a discussion of the optical properties of the laser and frequency modulation characteristics of the modulator.

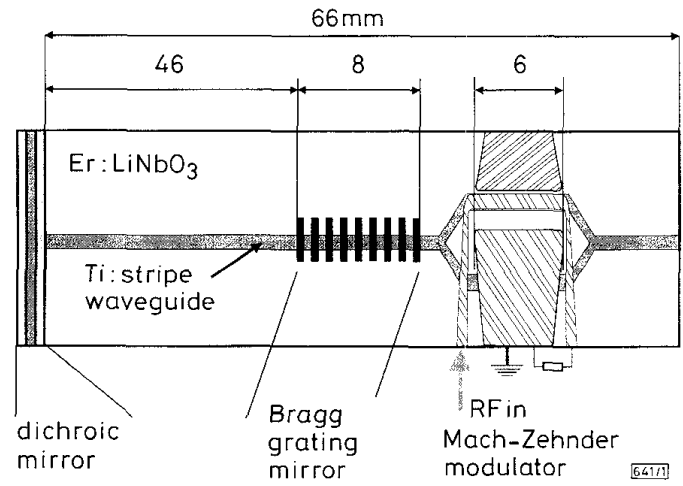

Fig. 1 Layout of integrated laser modulator sample

Sample configuration: Fig. 1 shows the layout of the laser/modulator configuration. The laser gain section between the $8 \mathrm{~mm}$ long Bragg grating and the dichroic input coupler mirror is $46 \mathrm{~mm}$ long. Additional amplification is provided by the $12 \mathrm{~mm}$ long erbium doped waveguide section behind the laser cavity where the MachZehnder waveguide interferometer is located.

Fabrication of the integrated laser/modulator device and characteristics of the components: The fabrication of the compact, single chip, source/modulator device involves the following steps:

i) Planar doping of a $\mathrm{Z}$ cut $\mathrm{LiNbO}_{3}$ wafer close to the surface by indiffusion of erbium at $1100^{\circ} \mathrm{C}$ for $100 \mathrm{~h}$ in an oxygen atmosphere.

ii) Transversal monomode stripe waveguide and Mach-Zehnder interferometer formation by a subsequent thermal diffusion of titanium stripes at $1030^{\circ} \mathrm{C}$ for $9 \mathrm{~h}$

iii) Dry etching of a $352.5 \mathrm{~nm}$ period Bragg grating to define one part of the compound DBR waveguide laser cavity: This surface corrugated reflector mirror is the key component allowing for monolithic integration with further components on the same chip. The first order DBR grating was holographically exposed in resist using a setup with beam collimating optics. This allows the recording of the two beam interference pattern of plane waves rather than spherical waves, and drastically reduces grating period chirp.
The resist pattern was then transferred $300 \mathrm{~nm}$ deep into the surface of $\mathrm{LiNbO}_{3}$ by dry etching techniques described in detail in $[4,5]$. The mirror shows a transmission drop exceeding $3.5 \mathrm{~dB}$ at the Bragg resonance, which indicates a high reflectivity of more than $50 \%$.

iv) Deposition of an $\mathrm{SiO}_{2}$ buffer layer of the travelling wave $\mathrm{Au}$ electrode outside the laser cavity on top of the Mach Zehnder structure.

v) End face deposition of a dielectric mirror stack of quarter wave layers of $\mathrm{SiO}_{2} / \mathrm{TiO}_{2}$ to complete the DBR laser cavity: The dichroic mirror shows a transmittance of $60 \%$ at the pump wavelength $(\lambda p$ $=1480 \mathrm{~nm})$ and simultaneously a high reflectance $(98 \%)$ at the laser emission wavelength $\left(\lambda_{s}=1561 \mathrm{~nm}\right)$.

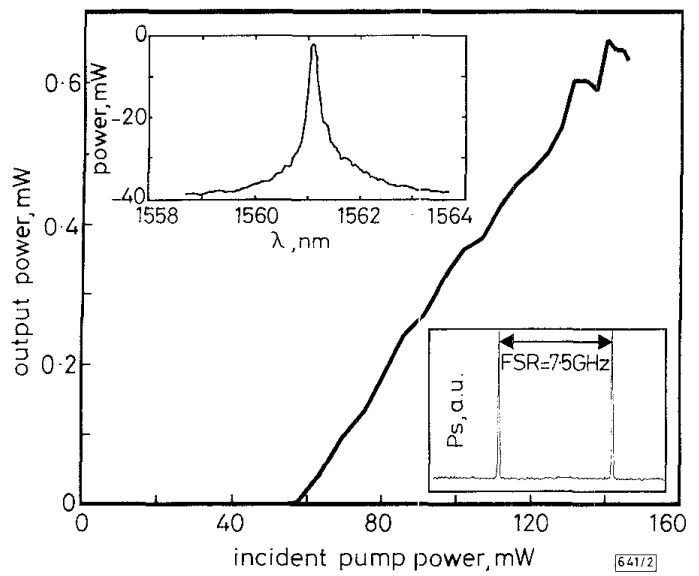

Fig. 2 Power characteristics, emission spectrum and longitudinal mode spectrum of integrated laser/modulator sample

Characterisation of the laser: The device, pumped with a commercial fibre pigtailed semiconductor laser diode, had a threshold of $54.8 \mathrm{~mW}$ incident pump power and a slope efficiency of $0.69 \%$ Fig. 2 shows the output power characteristics of the $54 \mathrm{~mm}$ long laser and the $12 \mathrm{~mm}$ long amplifier section. A maximum output power of $0.63 \mathrm{~mW}$ is obtained with $145 \mathrm{~mW}$ pump power under $(\sigma-)$ TE-polarised fibre butt coupling. The laser emission wavelength (see left inset of Fig. 2) of this specific device was adjusted by a properly selected Bragg grating period to $\lambda_{s}=1561.3 \mathrm{~nm}$, where Er: $\mathrm{LiNbO}_{3}$ has a local gain maximum. The small filter bandwidth of the Bragg mirror of $<0.2 \mathrm{~nm}$ results in stable single longitudinal mode emission. This has been confirmed by measurements with a scanning Fabry-Perot interferometer of $7.5 \mathrm{GHz}$ free spectral range (FSR) and a finesse of 200 (see right inset of Fig. 2).

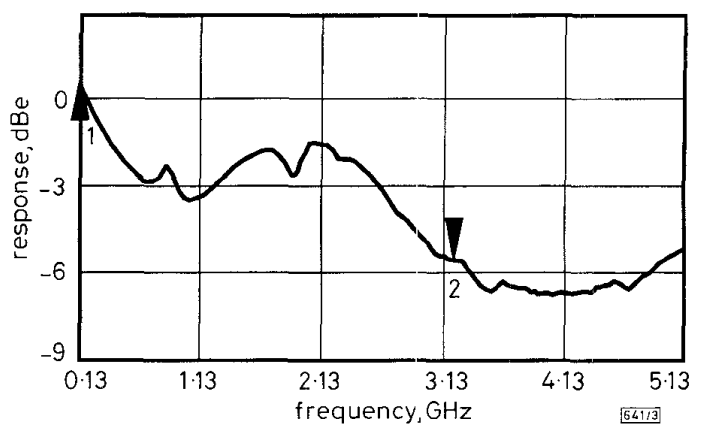

Fig. 3 Electro-optic bandwidth measurement of combined laser/modulator $-5.87 \mathrm{dBe}$ at $\Delta v=3.057 \mathrm{GHz}$

Characterisation of the modulator: The frequency response of the integrated laser/modulator device with a serial resistance of the electrode of $10 \Omega$ and the calculated $\mathrm{RF}$ impedance of $27 \Omega$ was measured with an HP 8703A lightwave component analyser. Fig. 3 shows the response under electro-optical modulation in lumped electrode configuration. The integrated laser/modulator device shows a bandwidth of $3.057 \mathrm{GHz}$ at $-6 \mathrm{dBe}$. Although designed for travelling wave operation (see Fig. 1) impedance matched surface mounted chip resistors could not be attached owing to the voltage 
requirements of more than $20 \mathrm{~V}$ needed to switch the modulator from the 'on' to the 'off' state. This high voltage for the first transmitter prototype is caused mainly by the short electrode length of $6 \mathrm{~mm}$ and the unfavourably low electro-optic coefficient $r_{31}$ exploited in this waveguide configuration ( $\mathrm{Z}$-cut $\mathrm{LiNbO}_{3}$, Ypropagation and TE-polarised emission wavelength).

Conclusion: We have demonstrated single frequency emission of an integrated optical DBR laser and an extracavity Mach-Zehnder type intensity modulator monolithically integrated on the same chip. Emission at $1561 \mathrm{~nm}$, with a threshold of $55 \mathrm{~mW}$ and a maximum output power as high as $0.63 \mathrm{~mW}$, was obtained for the combined laser/modulator device. The integrated fixed frequency light source and the intensity modulator shows a bandwidth of $3.1 \mathrm{GHz}$ at $-6 \mathrm{dBe}$.

Acknowledgments: This work has been supported by the EU and the Swiss BBW, under the EU RACE programme project R2013 EDIOLL and under the BBW contract No. $92.005 \mathrm{a}$.

(C) IEE 1996

Electronics Letters Online No: 19960602

11 March 1996

J. Söchtig, H. Schütz and R. Widmer (Paul Scherrer Institut Badenerstrasse 569, CH-8048 Zurich, Switzerland)

R. Corsini, D. Hiller, C. Carmannini, G. Consonni, S. Bosso and L. Gobbi (Pierlli Cavi, SpA, DBT/RST/COS, Viale Sarca 222, I-20126 Milnao, Italy)

\section{References}

1 BrinkmanN, R., SOHLER, $w$, and Suche, H.: 'Continuous-wave erbium-diffused $\mathrm{LiNbO}_{3}$ wave guide laser', Electron. Lett., 1991 27, pp. $415-416$

2 SUCHE, H., BAUMANN, I, Hiller, D., and SOHLER, W: 'Er:Ti:LiNbO ${ }_{3-}$ waveguide laser', Electron. Lett., 1993, 29, pp. 1111-1112

3 BAUMANN, I, JOHLEN, D., SOHLER, W, SUCHE, H., and TIEN. F.: 'Acoustically tunable Ti:Er:LiNbO 3 waveguide laser'. ECOC 94 , Florence, September 1994, (Post deadline paper)

4 SÖCHTIG, J., GROB, R., BAUMANN, I., SOHLER, W. SCHUTZ, H., and WIDMER, R.: 'DBR waveguide laser in erbium-diffusion-doped LiNbO $_{3}$, Electron. Lett., 1995, 31, (7), pp. 551-552

5 SÖCHTIG, J., SCHÜTZ, H., WIDMER, R., LEHMANN, HW, and GROR R: 'Grating reflectors for erbium-doped lithium niobate waveguide lasers'. Proc. SPIE Conf, Nanofabrication and Device Integration, 2213, pp. $98-107$

\section{Oxide-confined vertical-cavity laser with additional etched void confinement}

\author{
H. Deng and D.G. Deppe
}

\begin{abstract}
Indexing terms: Vertical cavity surface emitting lasers,
\end{abstract}
Semiconductor junction lasers

A vertical-cavity surface-emitting laser that incorporates oxide confinement with additional etched void photon confinement is described. A room-temperature continuous-wave threshold of $75 \mu \mathrm{A}$ for a $3.5 \mu \mathrm{m}$-diameter lateral device size is achieved.

The threshold current of vertical-cavity surface emitting lasers (VCSELs) has been decreased dramatically with the introduction of native oxide confinement $[1-6]$, and such devices can play a future role in low power optoelectronic interconnects. The native $\mathrm{Al}_{x} \mathrm{O}$, serves as a buried insulator in addition to yielding a large lateral refractive index step within the laser cavity from $\sim 1.6$ $\left(\mathrm{Al}_{x} \mathrm{O}_{y}\right)$ to $\sim 2.95$ (AlAs). Although the lateral index step occurs in only one or two thin layers within the cavity, when placed close to the active region it results in good lateral index confinement [2 4]. Before the oxide-confined VCSEL we demonstrated another fabrication process for achieving a buried lateral index step based on an etched void [7]. In that work we found that, despite the nearly planar geometry of the laser cavity, a thin etched void placed within the distributed Bragg reflector (DBR) stack results in significant reduction in the lateral mode size, similar to what occurs with the native oxide confinement. In this Letter we show how the etched void can be conveniently combined with the buried native oxide layer. Side by side comparisons show that increased optical mode confinement occurs along with a threshold reduction when the etched void is included. A minimum room-temperature $\mathrm{CW}$ threshold current of $75 \mu \mathrm{A}$ is measured for a $3.5 \mu \mathrm{m}$-diameter device.

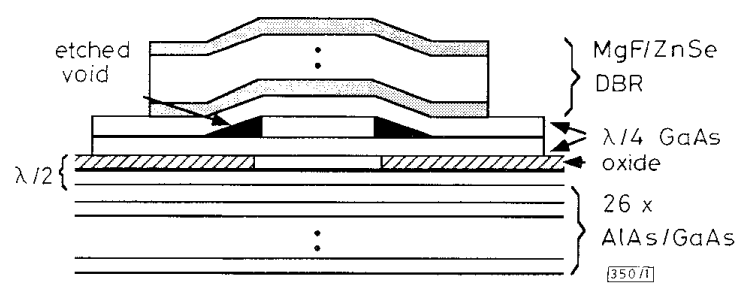

Fig. 1 Schematic cross-section of VCSEL structure with buried void confinement

A cross-section of the VCSEL employing both the etched void and native oxide is shown in Fig. 1. The semiconductor layers are grown by molecular beam epitaxy and from the GaAs substrate up include a 26-pair n-type AlAs/GaAs DBR, a half-wave cavity spacer of $\mathrm{Al}_{0.97} \mathrm{Ga}_{0.03}$ As with an $80 \AA$-thick $\mathrm{In}_{0.2} \mathrm{Ga}_{0.8} \mathrm{As} \mathrm{QW}$ in the centre, one quarter-wave pair of $p$-type GaAs and AlAs, and a $p$ type quarter-wave GaAs cap layer. The $80 \AA \operatorname{In}_{0.2} \mathrm{GA}_{0.8} \mathrm{As} \mathrm{QW}$ within the half-wave cavity spacer is clad on either side by undoped $100 \AA$-thick GaAs and $100 \AA$ undoped $\mathrm{Al}_{0.2} \mathrm{Ga}_{0.8}$ As layers. To process the etched void $15 \mu \mathrm{m}$-diameter $\mathrm{SiO}_{2}$ dots are defined on the wafer surface, and selective wet etching is used to expose the first $p$-type quarter-wave thick AlAs layer. The exposed AlAs is then selectively etched using a $1: 10 \mathrm{HCl}: \mathrm{H}_{2} \mathrm{O}$ solution to leave a $3.5 \mu \mathrm{m}$ AlAs post beneath the undercut GaAs top layer. During drying the liquid surface tension pulls the edge of the cap layer into contact with the GaAs layer below, resulting in a tapered void ring around the AlAs post (see Fig. 1). The void region is pink in colour and surface profiling shows the lateral width of the void ring to be $\sim 1.5 \mu \mathrm{m}$. To prevent the AlAs post from later oxidation, a rapid thermal anneal in forming gas $\left(10 \% \mathrm{H}_{2}\right.$ in $\left.\mathrm{N}_{2}\right)$ is performed on the sample at $450^{\circ} \mathrm{C}$ for $30 \mathrm{~s}$. This 'seals' the AlAs post against steam oxidation [8]. With the $\mathrm{SiO}_{2}$ and GaAs cap layers acting as a mask, the second GaAs layer is etched, exposing the upper $\mathrm{Al}_{0,47} \mathrm{Ga}_{0,03} \mathrm{As}$ half-wave cavity spacer layer, which is then steam oxidised at $450^{\circ} \mathrm{C}$ [1] leaving a $\sim 4 \mu \mathrm{m} \mathrm{Al}_{0.97} \mathrm{Ga}_{0.03} \mathrm{As}$ region. The self-aligned perimeters of the $\mathrm{AlAs}$ and $\mathrm{Al}_{0.97} \mathrm{Ga}_{0.03} \mathrm{As}$ regions overlap closely (see Fig. 1), with the top AlAs post slightly octagonally shaped due to the $1: 10 \mathrm{HCl}: \mathrm{H}_{2} \mathrm{O}$ etch. After removing the $\mathrm{SiO}$, and defining the metal contact, a five pair dielectric DBR of $\mathrm{MgF}_{2} / \mathrm{ZnSe}$ is deposited using electron beam evaporation. We also fabricate a comparison sample in the same manner as above but with an $\mathrm{HCl}: \mathrm{H}_{2} \mathrm{O}$ etch time to leave an upper AlAs post of $8 \mu \mathrm{m}$ diameter. This sample is oxidised simultaneously with the first to also define $4 \mu \mathrm{m}$ unoxidised $\mathrm{Al}_{0,97} \mathrm{Ga}_{003} \mathrm{As}$ region, and which removes the $8 \mu \mathrm{m}$-diameter etched void ring from the optical cavity.

With the five pair $\mathrm{MgF}_{2} / \mathrm{ZnSe} \mathrm{DBRS}$ the threshold currents measured on the etched void devices range from 106 to $130 \mu \mathrm{A}$, while the oxide-only devices ( $8 \mu \mathrm{m}$ etched void ring) range from 230 to $300 \mu \mathrm{A}$. Fig. 2 shows the lowest room temperature CW threshold current for the $3.5 \mu \mathrm{m}$ void confinement $(\sim 4 \mu \mathrm{m}$ unoxidised region) laser of $106 \mathrm{\mu A}$ with the differential slope efficiency of $34 \%$. The lasing wavelength is $9949 \AA$. For the comparison laser, Fig. 3 shows the lowest CW threshold current of $230 \mu \mathrm{A}$ with a slope efficiency of $31 \%$ and lasing wavelength of $9959 \AA$. The influence of the etched void region on the mode confinement is also studied by examining the far field radiation patterns [3]. The $1 / e^{2}$ width of the singlemode far field of the void confinement laser is measured to be $16.6^{\circ}$ (Fig. 2 inset), while that with the $4 \mu \mathrm{m}$ oxidised only region is $11.6^{\circ}$ (Fig. 3 inset). If a Gaussian mode size approximation is valid the far fields correspond to a $2.2 \mu \mathrm{m}$-diameter mode size for the etched void device against a 3.1 $\mathrm{m}$-diameter lasing mode for the oxide-only VCSEL. For the oxide-only VCSEL the present thresholds and far fields are close to those measured previously [3]. We estimate the radius of curvature of the upper DBR to be $\sim 30 \mu \mathrm{m}$, which is large compared to 